\section{Cahiers de Narratologie}

Analyse et théorie narratives

33 | 2018

L'Art du roman chez Umberto Eco

\title{
Malentendu et implicite : une nouvelle interprétation du film Balzac et la petite tailleuse chinoise
}

Wenjun Deng

\section{(2) OpenEdition}

Journals

Electronic version

URL: http://journals.openedition.org/narratologie/8363

DOI: $10.4000 /$ narratologie. 8363

ISSN: 1765-307X

Publisher

LIRCES

\section{Electronic reference}

Wenjun Deng, « Malentendu et implicite : une nouvelle interprétation du film Balzac et la petite tailleuse chinoise », Cahiers de Narratologie [Online], 33 | 2018, Online since 23 July 2018, connection on 15

November 2019. URL : http://journals.openedition.org/narratologie/8363; DOI : 10.4000/narratologie. 8363

This text was automatically generated on 15 November 2019.

Article L.111-1 du Code de la propriété intellectuelle. 


\title{
Malentendu et implicite : une nouvelle interprétation du film Balzac et la petite tailleuse chinoise
}

\author{
Wenjun Deng
}

\section{AUTHOR'S NOTE}

This article is supported by « the Fundamental Research Funds for the Central Universities »

1 Le cinéaste utilise sa caméra et son art pour enregistrer le temps présent et aussi pour revenir sur le passé. Le cinéma nous aide à comprendre le passé en se faisant une vision du monde et modifie également notre conception de la mémoire. En reconstituant un événement du passé, le cinéaste joint sa mémoire personnelle à la mémoire collective et porte son propre point de vue sur ce dernier. C'est ce que Marc Ferro précise, "par sa connaissance du présent, il [cinéaste] aide à comprendre ce qui a pu être le passé, puisque l'histoire est aussi bien le rapport entre passé et présent que ce qui, dans le présent, est l'héritage du passé ${ }^{1}$. Ainsi, nous nous permettons de dire que chaque mémoire individuelle est un point de vue sur la mémoire collective. Il n'y a aucun film politiquement neutre ou objectif : de l'élaboration du scénario à la construction des personnages, de la mise en intrigues de tels et tels événements au choix de l'instance narrative, une prise de vues est orientée même si ce n'est pas consciemment opéré. Dans le film Balzac et la petite tailleuse chinoise, le réalisateur Dai Sijie tourne son regard vers la communauté rurale pendant la Révolution culturelle et propose une vision du mouvement d'envoi des jeunes instruits à la campagne en rejoignant sa mémoire personnelle. A travers une analyse narratologique du film axée sur le narrateur, le point de vue et les personnages, cet article se consacre à l'interprétation des implicites conçus dans le film. Ceux-ci ont pour fonction d'incarner les points de vue du 
réalisateur vis-à-vis du monde rural et de l'idéologie de du mouvement de rééducation maoïste.

Avant de commencer cette analyse, nous montrons d'abord le malentendu qui existe vis-à-vis de cette œuvre dans les milieux intellectuels chinois. Le malentendu du public chinois concernant le roman Balzac et la petite tailleuse chinoise et son adaptation filmique n'est pas neuf. Malgré l'immense succès que le roman et son adaptation filmique ont connu en France, cette œuvre, paradoxalement, n'a pas attiré autant de regards de la part du public chinois. L'adaptation filmique n'a pas été autorisée à la diffusion en Chine continentale bien que le réalisateur Dai Sijie ait eu le permis de tourner sur le territoire chinois. Le traducteur du roman originellement rédigé en français, Yu Zhongxian, indique dans un article intitulé "L'influence de Balzac sur la petite tailleuse ${ }^{2}$ » publié à la suite de la traduction, que «la petite tailleuse dans le roman Balzac et la petite tailleuse chinoise représente la culture vernaculaire chinoise et elle est finalement séduite par la culture française représentée par Balzac. Ce qui satisfait largement la vanité et les valeurs du lecteur français ${ }^{3}$ \#. D'après lui, le thème de l'œuvre reste l'influence de Balzac sur la petite tailleuse chinoise et la conquête de la culture vernaculaire chinoise par la culture occidentale. Cette perception est aussi celle que la plupart des lecteurs chinois ont eue du roman. Pour eux, il s'agit d'un texte qui traite de la conquête culturelle et met l'accent sur la supériorité de la culture occidentale. L'apparition de ce malentendu n'est pas fortuite, étant donné que le roman a été écrit en langue française et publié en France, et que c'est l'écrivain français Balzac qui y exerce une influence profonde sur une jeune fille chinoise.

Le réalisateur Dai Sijie refuse de reconnaître l'interprétation que la plupart des lecteurs chinois donnent de son roman, qui consiste en la conquête de la culture orientale par la culture occidentale. Lorsque, au cours d'un entretien, il a été demandé pour quelle raison il avait conçu une telle histoire, il a révélé que «la vie était monotone dans la jeunesse de ma génération. Aimer lire est une chose naturelle. La littérature a apporté beaucoup de joie à ma vie à la campagne lors du mouvement d'envoi des jeunes instruits. À mon avis, la littérature est une chose superbe et romantique, ainsi j'ai voulu développer l'idée qu'un livre peut changer la vie ${ }^{4}$ ». Il existe dans les milieux intellectuels chinois des opinions différentes à l'égard de l'interprétation du roman. D'après la professeure Pan Wen ${ }^{5}$, Dai Sijie raconte de manière objective dans son roman une histoire de génération perdue au lieu d'une histoire de conquête culturelle ; il s'agit de l'enfermement des esprits, de la désorientation culturelle et du désir de lecture qui ont probablement eu lieu à cette époque-là. La cause en est que, explique-t-elle, « dans les années de la Révolution culturelle, tous ceux qui sont à la recherche des lumières sont exclus du savoir et de la culture - non seulement de la culture occidentale mais aussi de leur culture nationale ; la lecture clandestine les excite et même change leur destinée. Ils sont une génération spirituellement perdue. C'est la raison pour laquelle je préfère interpréter le roman comme une histoire sur l'errance spirituelle et l'aventure de la jeunesse ${ }^{6} »$.

Pour avoir une interprétation pertinente du film, il convient, en premier lieu, de tenir compte de l'expérience de Dai Sijie qui est aussi le réalisateur du film. En 1971, à l'âge de 17 ans, Dai a été envoyé dans un camp de rééducation dans un village reculé et difficile d'accès dans les montagnes de la province du Sichuan. Ses parents, qui étaient docteurs à l'époque, ont été condamnés comme « ennemis du peuple » et mis en prison. Après la fin de la Révolution culturelle, il a repris ses études en histoire d'art et obtenu 
une bourse à l'Institut des hautes études cinématographiques françaises en 1984, et depuis réside en France. C'est cette expérience personnelle dont il s'inspire pour l'écriture du roman Balzac et la petite tailleuse chinoise qu'il a mise en scène en 2002 à l'aide de cinéastes français. Nous pourrions ainsi dire que l'auteur raconte sa propre expérience de jeune instruit en joignant sa mémoire personnelle à la mémoire collective du mouvement d'envoi des jeunes instruits à la campagne, et que le personnage de Ma Jianling est l'incarnation parfaite de l'auteur lui-même.

5 En deuxième lieu, il convient de replacer le récit du film dans le contexte historique et social qu'il relate. Le mouvement d'envoi des jeunes instruits à la campagne se déclencha sous l'impulsion de Mao en 1968 pendant la Révolution culturelle et s'acheva en 1980. Pendant cette dizaine d'années, près de dix-sept millions de jeunes citadins ont été envoyés dans les régions rurales pour être rééduqués auprès des paysans. Confrontés à de dures réalités dans les régions reculées et pauvres et souffrant d'une double pauvreté, matérielle et spirituelle, les jeunes instruits perdirent leurs illusions et sombrèrent dans le désespoir. Les jeunes instruits subirent souvent la persécution des cadres locaux et des chefs de village, certaines jeunes instruites s'avilirent face à la tentation de ces derniers sous le prétexte de la possibilité d'un retour en ville. Ainsi est engendrée dans la Révolution culturelle la "génération perdue ", à laquelle Michel Bonnin a consacré un ouvrage intitulé Génération perdue : le mouvement d'envoi des jeunes instruits. À l'égard de la définition de cette " génération perdue ", il écrit :

"Ce sont en principe les trois promotions de collégiens et de lycéens des années 1966-1968, on les appelle "les trois vieilles promotions". Les membres de ces "les trois vieilles promotions" sont ceux qui ont le plus fort sentiment d'appartenance à un groupe spécifique, car leur expérience est la plus marquante : participation à la Révolution culturelle comme Gardes rouges, puis départ quasi général à la campagne dans des conditions souvent très dures et séjour particulièrement long puisque certains ont dû attendre dix ou onze ans pour revenir en ville, à la fin du mouvement, en $1979^{7}$ ».

Il existe dans la société chinoise une opinion publique, c'est que le mouvement d'envoi des jeunes instruits, auquel des millions de jeunes instruits ont sacrifié leurs illusions et leur jeunesse, voire leur vie, est effectivement une "duperie ». Ayant une expérience personnelle, quel avis et quels souvenirs les jeunes instruits ont-ils sur cet événement ? Considèrent-ils qu'ils aient été dupés? Pas vraiment. La marginalisation du déplacement des grandes villes aux régions rurales, pour certaines personnes n'est pas évidemment un "exil ", mais une "libération" dans un certain sens. Par exemple, Chen Kaige, ancien jeune instruit et cinéaste éminent de la Cinquième Génération, révèle dans un entretien : "J'ai été envoyé à la province du Yunnan pour recevoir une rééducation, on ne peut pas vraiment dire que j'y aie été obligé. Je désirais échapper à la discrimination et à la dépression, chercher mon idéal de vie heureuse dans la vaste terre ${ }^{8} »$. Il est naturel qu'il n'existe pas une mémoire unique pour tout événement d'importance. Par rapport au mouvement d'envoi des jeunes instruits, d'après Michel Bonnin, « deux sensibilités opposées traversent les mémoires diverses : la nostalgique et la critique. Rien n'est plus banal et universel que la nostalgie de la jeunesse, surtout quand cette jeunesse s'est déroulée dans une période exceptionnelle, laissant des souvenirs forts. Même les intellectuels les plus critiques sur la Révolution culturelle et le mouvement d'envoi à la campagne ont d'ailleurs des anecdotes personnelles agréables ou intéressantes à raconter sur cette époque ${ }^{9}$." En reconstituant cette expérience dans son film, quel point de vue l'auteur adopte-t-il ? Quelle sensibilité ou nostalgie lui reste-t-il? Quelle critique formule-t-il vis-à-vis de son expérience de 
rééducation ainsi que du monde rural où il a vécu sa jeunesse ? Le film est réalisé trente ans après le mouvement d'envoi des jeunes instruits, cet éloignement temporel permetil à l'auteur de modifier sa vision de cette expérience qu'il a vécue dans sa jeunesse quand il regarde en arrière trente ans plus tard? L'intention réelle du réalisateur consiste-t-elle à transmettre une idée de conquête culturelle? Ce sont autour de ces problématiques que cette analyse se déroulera.

\section{La coexistence de deux sensibilités : la critique et la nostalgie}

$7 \quad \mathrm{Au}$ lieu de ressusciter une mémoire traumatisante ou de mener une critique radicale de la «tromperie » du mouvement d'envoi des jeunes instruits, le film mène plutôt une critique de manière métaphorique et implicite sur l'idéologie du mouvement d'envoi des jeunes instruits. Le titre du film, Balzac et la petite tailleuse chinoise, est conçu de façon originale et allégorique. Les questions se posent : quel rapport entretient Balzac avec la petite tailleuse chinoise? Quelle histoire a eu lieu entre ce grand écrivain français et une petite tailleuse chinoise ? Le réalisateur associe certains signes culturels qui sont effectivement sans aucun rapport intrinsèque, tels que Mozart et le président Mao, Balzac et la petite tailleuse chinoise. C'est à travers cette interversion des signes culturels que le réalisateur implique de façon allégorique les troubles culturels pendant la Révolution culturelle. En plus, l'idéologie du mouvement d'envoi est mise en cause par le réalisateur à travers la mise en jeu d'une série de tensions dans les relations entre jeunes instruits et le chef du village, entre culture urbaine et culture rurale.

tension dans les relations entre les différents actants dans le film conçue par le réalisateur a pour fonction d'incarner ses points de vue vis-à-vis du monde rural et de l'idéologie du mouvement de rééducation maoïste, où coexistent la critique et la nostalgie. Algirdas-Julien Greimas a posé dans son livre Du Sens (1977) le «carré sémiotique » dans lequel il s'attaque directement au problème de la signification et postule qu'«il existe un modèle susceptible de décrire l'articulation du sens de façon universelle à l'intérieur de tout micro-univers sémantique ${ }^{10}$ ».

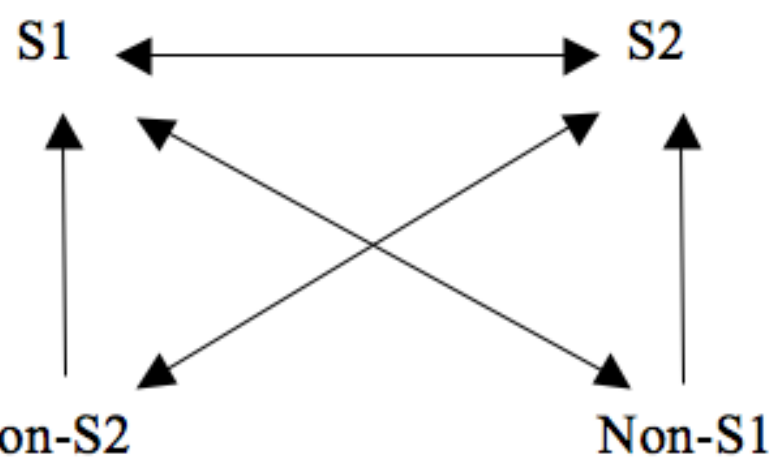

9 Ce schéma est largement utilisé dans l'analyse structurale du récit filmique et paraphrasé comme tel, "les termes de base $S_{1}$ et $S_{2}$ sont liés par une relation de contrariété (ce sont des contraires sémantiques, e.g. Bien et Mal) ; les termes non- $\mathrm{S}_{1}$ et non- $\mathrm{S}_{2}$ sont respectivement les opposés des deux premiers; les flèches marquent des relations de présupposition ${ }^{11} »$. Cela constitue une voie d'approche pertinente que nous 
utilisons pour interpréter toutes les nuances des tensions dans les relations entre les jeunes instruits et le monde rural, qui pourront être schématisées de façon suivante :

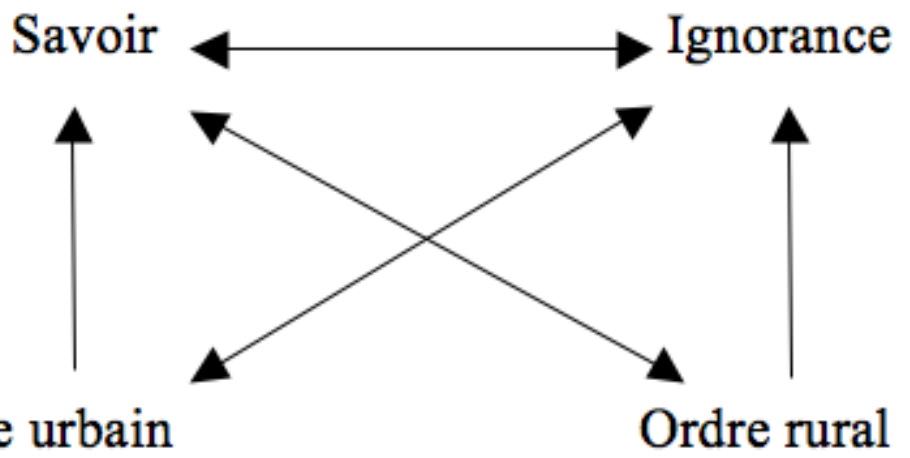
que cibles de la rééducation, ils sont à la fois opprimés par la politique du mouvement d'envoi et opposés à la communauté rurale d'accueil, il en découle ainsi que non-S1 sera «ordre rural», présupposant l' «ignorance» dans le film en question. Quant au contraire du "savoir », nous proposons de le définir comme "ignorance » représentée par la petite tailleuse et le chef du village alors que le dernier terme non-S2 sera défini comme « ordre urbain » qui présuppose le « savoir».

11 C'est à travers les nuances de la tension dans les relations entre ville et campagne que le réalisateur nous montre la situation à plusieurs niveaux : en premier lieu, c'est le statut d'opprimé des jeunes instruits dans la communauté rurale, révélé par la tension entre les deux jeunes instruits et le chef de village s'imposant d'emblée au tout début $\mathrm{du}$ film. Dès l'arrivée des deux jeunes instruits, Ma et Luo, au village, le chef de village examine et renverse leurs valises devant les villageois. Les deux jeunes instruits ont l'air mécontent mais n'osent dire un mot. Ayant découvert un livre, le chef demande à Luo d'en lire un paragraphe. Après avoir compris qu'il s'agissait d'un livre de cuisine, le chef le brûle et les sermonne sévèrement en déclarant : « les paysans révolutionnaires se laisseront jamais corrompre par une saleté de poulet bourgeois! Le président Mao vous a envoyé ici pour être rééduqués, pas pour faire des banquets réactionnaires. Dans notre montagne, on travaille dur et on mange du chou et du maïs ». La parole du chef révèle la condition matériellement pauvre du camp de rééducation. Ces épisodes présentent les jeunes instruits comme opprimés dans la communauté rurale d'accueil, ils mènent une vie matériellement et spirituellement pauvre. À travers cette tension entre les jeunes instruits et le chef de village, le réalisateur nous montre que les gens sont dans la quasi-impossibilité d'avoir accès aux livres et de parler de valeurs individuelles et de liberté, valeurs méprisées par le chef maoïste et détruites par la Révolution culturelle, durant laquelle la culture a connu des ravages sans précédent.

En deuxième lieu, c'est à travers la tension entre les jeunes instruits et le chef, récurrente dans le film, que le réalisateur nous montre l'illégitimité du leader maoïste pour mettre en cause l'idéologie du mouvement d'envoi des jeunes instruits. Tous les épisodes où la « foi » révolutionnaire du chef de village est en jeu sont traités sur le mode satirique et humoristique. Par exemple, le chef trouve le violon dans la valise de Ma. Le prenant pour un jouet, il demande à ce dernier de le brûler en accusant le violon d'être un objet bourgeois, mais Luo l'en empêche en déclarant : «On peut exécuter un morceau avec le violon, c'est "Mozart pense au président Mao" ». Prenant un air orgueilleux, le chef répond: "Mozart pense toujours au président Mao». En faisant 
confondre ces deux différents signes culturels qui n'ont aucun lien entre eux, l'auteur de la satire cherche à produire un effet comique et implique de façon allégorique les troubles culturels dans l'histoire chinoise à travers cette interversion culturelle. En même temps, le réalisateur révèle inconsciemment le point de vue des jeunes instruits vis-à-vis du monde rural et des paysans qui sont considérés comme ignorants aux yeux de ces derniers. De plus, lorsque le chef de village découvre que Ma raconte l'histoire du Comte de Monte-Cristo au vieux tailleur, il menace de les amener au bureau de dictature politique sous prétexte que Ma raconte une histoire réactionnaire. Luo aide Ma à sortir de cette situation épineuse en expliquant que «le personnage dans cette histoire est un pauvre marin. Selon la classification des classes du président Mao, il fait partie de la catégorie la plus révolutionnaire ». C'est également une autre façon d'affirmer implicitement la supériorité des jeunes instruits. Ils se jouent du chef en profitant de l'ignorance de ce dernier. Le caractère comique de ce personnage dote le film d'un style humoristique. Le réalisateur, en transformant une histoire tragique par son écriture personnelle, ôte à cette dernière la conscience de la tragédie et la représente même de manière comique. Le procédé permet de véhiculer les idées du réalisateur sur cette époque : bien qu'elle soit une expérience amère, Dai a vis-à-vis d'elle une attitude indulgente et il peut même l'interpréter de façon comique en l'observant avec le recul du temps.

13 En dernier lieu, le réalisateur affirme la supériorité des jeunes instruits en matière de culture et met en valeur le savoir à travers la tension dans les relations entre ville et compagne, entre savoir et ignorance. Le réveil, le violon et les romans défendus constituent dans le film les signes de la culture urbaine qui témoignent de l'impact de cette dernière sur la culture et la tradition rurale. Ce qui est mis en lumière par la narration du personnage en voix off: "Depuis toujours, les paysans réglaient leur activité sur le soleil. Cette coutume est remise en cause par l'arrivée de notre petit réveil ». Malgré leur statut politique d'opprimés, les jeunes instruits, grâce à leur supériorité en matière de savoir et de culture, incarnent consciemment ou inconsciemment le rôle de diffuseurs du savoir dans la communauté rurale. Ma et Luo, dans le film de Dai Sijie, incarnent ce rôle auprès des paysans incultes et ignorants à leurs yeux. Ainsi, le chef de village leur demande de raconter aux villageois le seul film de l'époque dont la diffusion est autorisée dans le bourg. Luo mélange intentionnellement les intrigues qu'il a lues dans les romans occidentaux lorsqu'il raconte le film aux villageois. C'est par ce moyen que la culture urbaine se diffuse parmi eux. Ici les jeunes instruits sont construits comme diffuseurs de la culture qui apportent des éléments de la culture urbaine, inconnue du monde rural, ainsi que des connaissances. C'est à travers cet épisode que la légitimité du projet politique maoïste de lancer le mouvement d'envoi des jeunes instruits à la campagne est mise en cause. Liu Xiaomeng, chercheur chinois sur le mouvement d'envoi des jeunes instruits, indique que :

"La doctrine de la "rééducation" mise en place par Mao est effectivement une récusation du système éducatif en vigueur pendant les dix-sept ans qui ont précédé la Révolution culturelle. La relation naturelle entre intellectuels et ouvriers ou paysans a été définie comme celle entre éduqué et éducateur. Sous la direction de cette doctrine, le mouvement de "monter à la montagne" et d'"aller à la campagne" est considéré comme un mouvement de réforme des personnes. On demande aux jeunes instruits de considérer des paysans comme des modèles de vie, de culture, de pensée et de moralité12 ${ }^{12}$. 
14 puisqu'ils sont ignorants aux yeux des jeunes instruits et du réalisateur lui-même. Il nous montre aussi que ce sont les jeunes instruits, qui possèdent des connaissances, qui deviennent «éducateurs » pour instruire les gens ignorants. Ainsi, le projet politique de la Révolution culturelle est inversé dans son fondement même. La sensibilité complexe du réalisateur vis-à-vis de cette expérience de rééducation est révélée : d'une part, il met en cause la légitimité du mouvement alors qu'il essaie, d'autre part, de nous convaincre que cette expérience n'est pas tout à fait inutile. construit. Celui-ci tire sa force de conviction de sa propre expérience et l'organisation de l'intrigue a pour fonction d'incarner les idées du réalisateur sur le mouvement d'envoi des jeunes instruits à la campagne, qui, d'après lui, n'est pas tout à fait inutile. Ce message est transmis par la mise en intrigue des expériences des deux jeunes instruits dans leur vie quotidienne à la campagne. Après avoir réussi à plomber une dent au chef, Luo est surnommé " Docteur Luo " par les villageois qui font appel à lui quand ils sont malades. Le réalisateur nous montre à la fin du film que Luo est réellement devenu docteur et sa réussite est mise en évidence par la conversation entre Ma et l'épouse de Luo. Ma demande à Luo : « Tu es devenu un dentiste réputé ? " Luo rit sans répondre. L'épouse de Luo répond: "Oui. Il est aussi professeur titulaire et directeur de thèses. C'est une sommité nationale ». À travers cet épisode, le réalisateur tente de nous convaincre d'une chose : l'expérience de rééducation n'a pas eu un effet complètement destructeur sur la vie des jeunes instruits. Comme évoqué au début, Michel Bonnin indique que la nostalgie et la critique constituent deux sensibilités opposées qui traversent les mémoires diverses concernant l'expérience de rééducation. La nostalgie de la jeunesse est une chose naturelle, surtout d'une jeunesse passée dans une période exceptionnelle qui laisse des souvenirs très forts et profonds. Cela nous permet de comprendre que Dai Sijie, ancien jeune instruit, révèle consciemment ou inconsciemment une certaine nostalgie dans son film.

Ce sentiment nostalgique que le réalisateur porte vis-à-vis de son vécu de rééducation est révélé par l'utilisation de la narration à la première personne. Le narrateur s'introduit dans le monde diégétique par la narration en voix off : «La première fois que j'ai pris ces escaliers de montagne remonte en 1971 avec Luo Ming. Je n'ai jamais autant marché. De la ville de Tian Quan à la montagne du Phénix où nous étions envoyés en rééducation, il y avait deux jours de marche ». Nous pouvons très vite identifier cette voix comme étant celle de l'un des protagonistes du film, qui sont les jeunes instruits envoyés à la montagne du Phénix. Au début du film, ce personnage, Ma Jianling, annonce son rôle de narrateur d'une histoire ayant eu lieu dans sa jeunesse. Outre sa fonction narrative, qui est une fonction essentielle, le narrateur a une fonction "émotive " selon l'appellation de Jacobson. Même si cette dénomination, d'après Gérard Genette, est malencontreuse, il indique que "c'est celle qui rend compte de la part que le narrateur, en tant que tel, prend à l'histoire qu'il raconte, du rapport qu'il entretient avec elle : rapport affectif, certes, mais aussi bien moral ou intellectuel, qui peut prendre la forme d'un simple témoignage, comme lorsque le narrateur indique la source d'où il tient son information, ou le degré de précision de ses propres souvenirs, ou les sentiments qu'éveille en lui tel épisode ${ }^{13}$ ». Dans ce film, le narrateur-personnage entretient avec l'histoire qu'il raconte un "rapport affectif » et assume une fonction «émotive » en puisant dans ses propres souvenirs. Cela permet de révéler le sentiment 
nostalgique que le réalisateur tente d'exprimer dans son film en se rappelant sa propre expérience de jeune instruit.

17 La sensibilité nostalgique est aussi mise en évidence par le changement de stratégie narrative à la fin du film. Dans le livre Esthétique du film, il est indiqué que la fonction du narrateur consiste à choisir, pour la conduite de son récit, parmi un certain nombre de procédures dont il n'est pas nécessairement le fondateur, mais bien plus souvent l'utilisateur. Toujours selon les auteurs du livre, la notion de narrateur n'exclut pas pour autant l'idée de production et d'invention : «le narrateur produit bel et bien à la fois un récit et une histoire, de même qu'il invente certaines procédures du récit ou certaines constructions d'intrigue ${ }^{14} »$. Dans le film, le narrateur brise la linéarité de la narration à la fin du film, qui est interrompue par la visualisation du présent accompagnée par la voix off de Ma: «À cette époque, je me demande pourquoi je n'avais pas envisagé ce qui nous arrivait. Depuis quinze ans, je mène ma carrière en France. J'ai joué dans des orchestres à Lyon et à Toulouse, puis avec des amis, j'ai fondé un quatuor à Paris pour interpréter Mozart et Beethoven, mes compositeurs favoris ». La narration du narrateur nous permet de nous repérer temporellement dans l'espace diégétique, quinze ans après la fin du mouvement. Ce changement d'espace et de temps brusque à la fin du film, qui semble très surprenant, est mis en cause au sein de la communauté des spectateurs chinois. À mon avis, c'est une belle invention du récit pour mettre en lumière le sentiment nostalgique du personnage qui renvoie au réalisateur lui-même. En regardant un reportage télévisuel français sur la construction des Trois Gorges, Ma apprend que les villages aux alentours de la montagne du Phénix seront submergés du fait des travaux, ce qui l'amène à retourner à la montagne pour $\mathrm{y}$ chercher la petite tailleuse. C'est à travers la tentative du personnage de retrouver sa jeunesse perdue que le réalisateur met en lumière son sentiment nostalgique vis-à-vis de sa jeunesse passée à la campagne. En retournant en Chine et rejoignant Luo à Shanghai, Ma lui montre la vidéo qu'il a prise dans la montagne du Phénix : la maison de la petite tailleuse, la grotte où ils cachaient les romans défendus, le village où ils ont habité pendant la période de rééducation, et le chef du village qui est devenu très vieux. C'est dans cet épisode qu'est révélé le changement du point de vue des personnages vis-à-vis du monde rural. Quand les personnages se rappellent leur expérience de rééducation, leur tristesse et leur déception sont remplacées par la nostalgie du temps passé et le regret du départ de la petite tailleuse.

Le film est sorti en 2001, trente ans après que le réalisateur du film ait été envoyé à la campagne. Ce laps de temps permet au réalisateur d'avoir une perception de son expérience de jeune instruit en mutation continuelle par rapport à sa première expérience en 1971. C'est ce que Wallace Martin précise, « il existe deux variables dans l'autobiographie qui permettent d'éviter de présenter une image immuable de la vie de l'écrivain. La signification des événements peut changer quand ils sont observés avec le recul ; et le soi-même qui décrit les événements pourrait avoir changé dès que ceux-ci sont originairement vécus ${ }^{15}$ ». Les ombres du traumatisme de la Révolution culturelle se dispersent au fur et à mesure du temps qui passe. Le réalisateur dote le dénouement du film d'une valeur métaphorique: la submersion de la montagne du Phénix par l'écoulement de l'eau suite aux travaux des Trois Gorges signifie implicitement que les souvenirs intimes qui appartiennent à ces trois jeunes sont submergés par le temps qui s'écoule. En reconstituant cette expérience sur le grand écran, le réalisateur révèle son attitude généreuse et indulgente vis-à-vis de cette expérience de rééducation au lieu d'en faire une critique radicale ou d'exprimer son angoisse d'avoir été «dupé ». Nous 
pouvons remarquer l'évolution de son point de vue sur le monde rural, introduit par celui des personnages. Même s'il s'agit de la mémoire traumatisée d'une génération sacrifiée, elle a pu être représentée de manière romantique, parce qu'il s'agit malgré tout d'une histoire de jeunesse.

\section{La mise en exergue des valeurs individuelles}

19 En révélant de façon implicite son point de vue vis-à-vis du mouvement d'envoi des jeunes instruits, Dai Sijie met l'accent dans son film sur la liberté individuelle et les valeurs individuelles à travers la construction du personnage de la petite tailleuse. Le personnage, d'après François Vanoye, est appréhendé comme une personne ou plutôt une représentation, « un "signe" global lui-même constitué de signes dessinant un rôle, un type, une fonction, une absence quelquefois ${ }^{16} »$. Ce sont les énoncés descriptifs, narratifs, dialogués qui nous informent, de façon plus ou moins lacunaire selon les nécessités du récit, sur l'identité, les traits physiques et psychologiques du personnage, son action sur les autres ainsi que son évolution. Le réalisateur légitime ses idées à partir de son expérience personnelle et construit un récit qui tire sa force de conviction de son expérience, mais dont les personnages sont fictifs et dont l'organisation a pour fonction de véhiculer les idées du réalisateur sur la valeur de l'individu et de la liberté.

La petite tailleuse est l'héroïne du film, construite au début du film comme une campagnarde innocente. Faute d'instruction, elle est ignorante mais curieuse de tout. Le changement de ce personnage central est conçu comme une ligne directrice du récit du film, qui a pour objet d'incarner cette idée du réalisateur : la lecture modifie parfois le destin de l'individu et permet aux gens d'avoir une conscience individuelle. La découverte des romans défendus constitue une intrigue cruciale dans le système de causalité du récit, en apportant à la fois un rayon de soleil dans l'existence des jeunes instruits mais aussi en éclairant le monde spirituel de la petite tailleuse. L'évolution de cette dernière est mise en lumière notamment par les remarques des autres personnages, par exemple par les mots du vieux tailleur. Il dit à Luo : « Ma petite fille a changé. Le jour où je suis rentré à la maison ", la narration du vieux tailleur laisse place à un retour en arrière : la petite tailleuse montre son soutien-gorge à ses amies en expliquant : "Ça s'appelle un soutien-gorge, pour mettre en valeur la poitrine. On dit comme ça dans les livres "civilisés"». " Ça signifie quoi, "civilisé" », questionne en voixoff une de ses amies. La petite tailleuse répond sérieusement : "L'homme sauvage n'a que des sentiments. L'homme civilisé a des sentiments et des idées ». Le flash-back laisse place à la visualisation du présent. Le vieux reprend la parole : «Je n'ai jamais tremblé. Un livre peut parfois changer la vie des gens ». Dans un autre épisode où Luo revient à la montagne après une séparation de deux mois avec la petite tailleuse, un plan d'ensemble nous le montre dans la maison du tailleur, assis à table, en compagnie de la tailleuse, son grand-père et Ma.

$21 \mathrm{Ma}$ (dit à Luo): Tu te souviens ? Tu avais juré de la sortir de son ignorance. C'est réussi. Luo : $\mathrm{Tu}$ as entendu comment elle parle ? Elle n'a même plus d'accent.

$\mathrm{Ma}$ : Tout ça, c'est grâce à nous.

Luo : ça a servi de lui lire des romans.

22 Cette conversation montre que la lecture des romans de Balzac transforme radicalement la jeune fille qui n'est plus une innocente paysanne; elle devient une femme épanouie qui désire découvrir par elle-même la vie en dehors des montagnes. 

changement signale l'évolution de la posture des personnages au cours du récit. Au début du film, la petite tailleuse est vêtue comme une montagnarde, elle porte une chemise rouge de modèle traditionnel et natte ses cheveux en tresses très longues alors que nous remarquons un changement remarquable de l'habillement de ce personnage dans la dernière séquence $d u$ film. Un jour, arrive chez Ma et Luo le vieux tailleur essoufflé d'avoir couru pour les informer du départ de sa petite fille. Nous remarquons le changement de cette jeune fille : elle a fait couper ses cheveux et a changé pour une coiffure courte avec une petite décoration. Elle porte une chemise à manches courtes boutonnée dans le dos, avec un col à revers large et des motifs, et aux pieds, elle a des chaussures de tennis blanches.

\section{La petite tailleuse : Balzac.}

La caméra suit de dos la petite tailleuse qui s'éloigne petit à petit alors que Luo rejoint Ma.

Ma : Elle est partie?

Luo : Oui, à cause de Balzac. Elle dit qu'elle a appris une chose, la beauté féminine est un trésor inestimable.

Ces épisodes où le changement de la petite tailleuse est en jeu ont pour fonction d'exprimer les idées du réalisateur sur la liberté individuelle, largement mise en valeur dans la culture occidentale mais méprisée voire détruite par la révolution maoïste dans la société chinoise. S'inspirant de valeurs individuelles occidentales apprises dans les romans de Balzac, la petite tailleuse est désireuse de découvrir le monde extérieur. Le départ de cette petite montagnarde révèle sa recherche déterminée de liberté et sa valeur individuelle de femme malgré sa séparation d'avec son amant et son grand-père, et malgré la contrainte idéologique de l'époque qui impose l'enfermement de l'esprit dans les valeurs de la collectivité. Le réalisateur montre que les gens ont le droit d'accomplir leur destinée contre les règles du groupe et que les valeurs individuelles occidentales qui sont portées par la littérature ont une portée universelle.

\section{Conclusion}

En joignant la mémoire individuelle à la mémoire collective, Dai Sijie raconte apparemment une histoire concernant la jeunesse et l'amour, une surface sensible où la mise en cause du mouvement maoïste s'inscrit implicitement. La représentation de ces relations de tension a fonction de charge critique contre la méprise et la destruction des valeurs et de la liberté des individus dans les années de troubles de la Révolution culturelle. Aucun réalisateur n'avait porté autant d'intérêt à l'influence des jeunes instruits sur le monde rural. Dai construit les jeunes instruits comme diffuseurs du savoir dans le monde rural, qui se passionnent à raconter des films aux villageois et à lire des romans de Balzac à la petite tailleuse. La conception des relations tendues entre les jeunes instruits et le chef de village, entre culture urbaine et culture rurale a pour fonction, d'un côté, d'impliquer le point de vue des jeunes instruits vis-à-vis du monde rural et des paysans considérés comme ignorants, remettant ainsi en cause l'idéologie de rééducation, et d'un autre côté, d'affirmer l'idée du réalisateur que la lecture donne lieu à la prise de conscience de la valeur individuelle et la liberté individuelle, qui sont largement mises en valeur dans l'univers occidental.

Cahiers de Narratologie, 33 | 2018 
Wallace Martin souligne que «les autobiographes sont aussi faillibles que les autres êtres humains. Ils se présentent eux-mêmes sous la meilleure perspective en dissimulant certains faits, à défaut de reconnaître l'importance des autres et d'oublier les événements importants que les biographes peuvent montrer ${ }^{17}$ ». De même, dans cette écriture personnelle de l'expérience de jeunes instruits, Dai reconstitue les événements du passé de manière lyrique et dissimule consciemment ou inconsciemment certains faits importants, tels que la séparation familiale entraînée par le mouvement, le harcèlement des chefs de village sur les jeunes instruites sous prétexte de leur offrir l'opportunité du retour en ville ainsi que l'inadaptation et la marginalisation des jeunes instruits lorsqu'ils regagnent leurs villes d'origine. De fait, la mise en scène ne fait pas fonction de recherche historique, le réalisateur nous oriente pour lire la situation depuis sa propre perspective au lieu de montrer une fresque du mouvement d'envoi des jeunes instruits.

Cette génération perdue est la victime du culte et de l'idéalisme maoïste et leur cruelle aventure rurale leur apprend le réalisme, ce qui leur permet de se rendre compte de l'existence réelle des Chinois et de mener une réflexion profonde sur le régime socialiste et l'idéologie des autorités. Wang Xizhe, chercheur chinois, écrit que «la Révolution culturelle a eu pour effet secondaire la production d'une "génération réfléchie", engendrée dans un contexte historique et social spécifique. Riche d'une expérience sociale et politique exceptionnelle, cette génération a su développer un esprit critique quant à l'idéologie officielle ${ }^{18} »$. Le mouvement d'envoi de jeunes instruits est le produit de la Révolution culturelle. Au fur et à mesure que celle-ci se finit, des millions de jeunes instruits "exilés" regagnent leurs villes d'origine. Pourtant, la fierté à l'égard du passé peut être considérée, comme l'écrit Michel Bonnin, "comme un moyen d'affirmer une identité profondément menacée, comme un réconfort indispensable pour une génération sacrifiée ${ }^{19} "$; mais elle ne peut toutefois compenser la frustration de cette génération sacrifiée qui a été marginalisée par l'ordre urbain après quelques années de rééducation à la campagne. Face à l'inadaptation à la vie réelle lors du retour en ville, les jeunes instruits ne peuvent s'empêcher d'extérioriser leur sentiment nostalgique en se rappelant leur jeunesse passée à la campagne, même si ce sentiment nostalgique se double inévitablement d'une critique.

\section{NOTES}

1. Marc Ferro, Cinéma, une vision de l'Histoire, Paris, Éditions du Chêne, 2003, p. 162.

2. Yu Zhongxian, «Baer zhake dui xiaocaifeng de yingxiang " (L'influence de Balzac sur la petite tailleuse), postface de la traduction du roman Balzac et la petite tailleuse chinoise, Pékin, Beijing shiyue wenyi chubanshe (Éditions littérature et art d'octobre de Pékin), 2003. Cet article a été publié le $1^{\text {er }}$ août 2003 sur le site «Le vendredi de la culture » (Wenhua xingqi wu), Cf. http:// www.china.com.cn/chinese/feature/377574.htm, consulté le 21.02.2013.

3. Yu Zhongxian, "L'influence de Balzac sur la petite tailleuse ", postface de la traduction de Balzac et la petite tailleuse chinoise, Pékin, Beijing shiyue wenyi chubanshe (Éditions littérature et art d'octobre de Pékin), 2003. 
4. Cet entretien a été publié le 9 septembre 2003 sur le site Enorth (Beifang Wang), Cf. http:// ent.enorth.com.cn/system/2003/09/09/000630164.shtml, consulté le 21.02.2018.

5. Professeure de l'École du parti communiste de la province de Zhejiang.

6. Pan Wen, Tamen shi wenhua mimang er wuzhuo de yidai-Ping «Baerzhake yu zhongguo xiaocaifeng » de zhuti (Ils sont la génération de la perplexité et de la désorientation culturelle Le thème de Balzac et la petite tailleuse), Zhonggong zhejiang shengwei dangxiao xuebao (Le journal académique de l'Ecole du parti communiste de la province du Zhejiang), n 3, 2004, p. 122.

7. Michel Bonnin, «Une génération vraiment perdue ? » Préface du roman de Zhang Kangkang, L'impitoyable, Paris, Éditions Bleu de Chine, 1997, p. 6. Zhang Kangkang est une écrivaine chinoise née à Hangzhou en 1950 dans une famille d'intellectuels. Elle fait partie de la catégorie de jeunes instruits des «trois vieilles promotions». Elle a vécu huit ans dans le Nord de la Mandchourie, expérience décrite dans « L'Impitoyable ».

8. Luo Xueying, "Yingmu shang de zhuimeng ren » (Le chercheur de rêves sur le grand écran Entretien avec Chen Kaige), Yang Yuanying, Pan Ye, Zhang Zhuan, 90 niandai de diwudai (La Cinquième Génération dans les années 1990), Pékin, Zhongguo chuanmei daxue chubanshe (Éditions de l'Université de média de Chine), 2000, p. 277.

9. Michel Bonnin, « L'histoire de la Révolution culturelle et la mémoire de la "génération perdue" sont-elles condamnées à l'oubli? », Perspectives chinoises, n 4, 2007, p.65.

10. Jacques Aumont, Michel Marie, L'analyse des films, Paris, Éditions Nathan, 1999, p.102.

11. Ibid.

12. Liu Xiaomeng, Zhongguo zhiqing shi. Dachao (1968-1980) (Histoire des jeunes instruits chinois. La grande marée, 1968-1980), Pékin, Zhongguo shehui kexue chubanshe (Éditions des sciences sociales chinoises), 1998, p. 414.

13. Gérard Genette, Figures III, collection Poétique, Éditions du Seuil, 1972, p. 262.

14. Jacques Aumont, Alain Bergala, Michel Marie, Marc Vernet, Esthétique du film, Paris, Éditions Armand Colin, 2004, p. 78.

15. Wallace Martin, Wallace Martin, Recent Theories of Narrative, New York, Cornell University Press, 1986, P. 76. Texte original en anglais : There are two variables in autobiography that can keep it from presenting an unchanging picture of the writer's life. The significance of the events may change when they are viewed in retrospect ; and the self that describes the events may have changed since they were first experienced.

16. Francis Vanoye, Récit écrit, récit filmique, Paris, Éditions Nathan, 2002, p.117.

17. Wallace Martin, Recent Theories of Narrative, New York, Cornell University Press, 1986, p. 75. Texte original en anglais: autobiographers are as fallible as other human beings. They often present themselves in the best possible light, suppressing some facts, failing to recognize the significance of others, and forgetting incidents that biographers can show were important.

18. Michel Bonnin, «L'histoire de la Révolution culturelle et la mémoire de la "génération perdue" sont-elles condamnées à l'oubli ? ", Perspectives chinoises, n 4, 2007, p. 55.

19. Ibid., p. 65.

\section{ABSTRACTS}

The misconception of the Chinese public on the novel by Dai Sijie Balzac and the Little Chinese Seamstress (2000) and its film adaptation (2002) has been around for many years. In the film, Dai 
plots a series of tensions in the relations between the educated youth and the rural world during the Down to the Countryside Movement to implicitly depict his views on the rural world and on the ideology of the Maoist movement, involving both criticism and nostalgia of this experience. The relations between the educated youth and the rural world will be analyzed by the "Semiotic Square » of Algirdas Julien Greimas, which seems a relevant approach. By the construction of the character of the little seamstress, Dai Sijie reveals implicitly also his emphasis on the individual freedom and individual values. This article is devoted to the interpretation of implicates in the movie which helps us to understand the history and offers us an angle to observe the Chinese society during that period.

Le malentendu du public chinois quant au roman de Dai Sijie Balzac et la petite tailleuse chinoise (2000) et à son adaptation filmique homonyme (2002) n'est pas neuf. Dai développe dans le film une série des tensions dans les relations entre les jeunes instruits et le monde rural qui ont pour fonction d'incarner de manière implicite ses points de vue vis-à-vis du monde rural et de l'idéologie du mouvement d'envoi des jeunes instruits à la campagne, où coexistent la critique et la nostalgie. Les relations entre les jeunes instruits et le monde rural seront interprétées selon le « carré sémiotique » d'Algirdas-Julien Greimas, qui constitue une voie d'approche pertinente. Dai Sijie met également l'accent de façon implicite sur la liberté individuelle et les valeurs individuelles à travers la construction du personnage de la petite tailleuse. Cet article est consacré à l'interprétation des implicites mis en jeu dans le film qui rend intelligible cette histoire du passé et propose une vision de la société chinoise de l'époque.

\section{INDEX}

Chronological index: XXème siècle Geographical index: Chine

Mots-clés: Dai Sijie, Balzac et la petite tailleuse chinoise, implicite, nostalgique, critique

Keywords: Dai Sijie, Balzac and the Little Chinese Seamstress, implicate, nostalgia, criticism

\section{AUTHOR}

\section{WENJUN DENG}

Professeure à l'Université Jinan, Chine.

Axe de recherche actuel : le cinéma et l'histoire ; ancienne docteure en Histoire culturelle à

l'école doctorale Arts et Médias, Université Paris 3 ; son orientation de recherche consiste en les questions de mémoire et identité, et la représentation des traumatismes à l'écran. Sa thèse, intitulée « La mémoire collective de la Révolution culturelle dans le cinéma chinois contemporain », a été soutenue le 14 juin 2013 avec la mention « Très honorable avec félicitations ». 